Trinity University

Digital Commons@ Trinity

Mathematics Faculty Research

Mathematics Department

2004

\title{
Asymptotic Solutions of a Discrete Schrödinger Equation Arising from a Dirac Equation with Random Mass
}

Bernd Aulbach

Saber Elaydi

TrinityUniversity, selaydi@trinity.edu

Klaus Ziegler

Follow this and additional works at: https://digitalcommons.trinity.edu/math_faculty

Part of the Mathematics Commons

\section{Repository Citation}

Aulbach, B., Elaydi, S., \& Ziegler, K. (2004). Asymptotic solutions of a discrete Schrödinger equation arising from a Dirac equation with random mass. In B. Aulbach, S. Elaydi, \& G. Ladas (Eds.), Proceedings of the Sixth International Conference on Difference Equations, Augsburg, Germany 2011: New Progress in Difference Equations (pp. 349-358). Boca Raton, FL: CRC Press.

This Post-Print is brought to you for free and open access by the Mathematics Department at Digital Commons @ Trinity. It has been accepted for inclusion in Mathematics Faculty Research by an authorized administrator of Digital Commons @ Trinity. For more information, please contact jcostanz@trinity.edu. 


\title{
Asymptotic Solutions of a Discrete Schrödinger Equation Arising from a Dirac Equation with Random Mass
}

\author{
BERND AULBACH \\ Department of Mathematics, University of Augsburg \\ D-86135 Augsburg, Germany \\ E-mail: aulbach@math.uni-augsburg.de \\ SABER ELAYDI \\ Department of Mathematics, Trinity University \\ San Antonio, Texas 78212, USA \\ E-mail: selaydi@trinity.edu \\ and \\ KLAUS ZIEGLER \\ Department of Physics, University of Augsburg \\ D-86135 Augsburg, Germany \\ E-mail: ziegler@physik.uni-augsburg.de
}

\begin{abstract}
For a Dirac particle in one dimension with random mass, the time evolution for the average wavefunction is considered. Using the supersymmetric representation of the average Green's function, we derive a fourth order linear difference equation for the low-energy asymptotics of the average wavefunction. This equation is of Poincaré type, though highly critical and therefore not amenable to standard methods. In this paper we show that, nevertheless, asymptotic expansions of its solutions can be obtained.
\end{abstract}

Keywords Dirac particle in one dimension with random mass, Green's function, Poincaré type difference equation, Asymptotic expansion

AMS Subject Classification 34L40, 39A10, 39A11, 60H10 


\section{Introduction}

A Dirac particle is considered which can move only in one dimension. It is characterized by a two-component spinor wavefunction. The mass of the particle varies randomly in space. Such a random mass term can be the result of an interaction of the particle with an external random field that affects the kinetic properties. We are interested in the average wavefunction, averaged with respect to the random mass. Therefore, we have to evaluate the average Green's function which describes the evolution of the average wavefunction. A supersymmetric representation of the average Green's function is employed to perform the calculation. It allows us to translate formally the original one-dimensional continuous Dirac Equation with random mass into a discrete deterministic Schrödinger Equation. This equation is a fourth order linear difference equation of Poincaré type, i.e. an equation of the form

$$
x_{n+2}+p_{1}(n) x_{n+1}+p_{2}(n) x_{n}+p_{3}(n) x_{n-1}+p_{4}(n) x_{n-2}=0
$$

where the coefficients $p_{1}(n), \ldots, p_{4}(n)$ converge to constants $p_{1}^{*}, \ldots, p_{4}^{*}$, respectively, as $n \rightarrow \infty$. For this kind of equations there is a well developed classical theory (see e.g. Elaydi [7, Chapter 8]) providing information on the asymptotic behavior of the solutions. The basic results of the classical theory, however, assume that the four complex roots of the corresponding characteristic equation

$$
\lambda^{4}+p_{1}^{*} \lambda^{3}+p_{2}^{*} \lambda^{2}+p_{3}^{*} \lambda+p_{4}^{*}=0
$$

have pairwise distinct moduli. This assumption, however, turns out to be drastically violated by the Schrödinger equation arising in this paper. In fact, here the characteristic equation has 1 as a fourfold root.

The way we treat the Schrödinger equation in this paper is to first exploit the particular structure of this equation in order to reduce its order from four to two. The resulting second order equation is of Poincaré type, too, and the roots of the corresponding characteristic equation are +1 and -1 . They still have the same modulus, however, for this kind of problems there is a general asymptotic theory (even for higher order equations) based on the work of Birkhoff [3, 4], Birkhoff and Trjitzinsky [5] and Adams [1]. Since in our setting the reduced equation is of second order, we can use a simplified version of the general theory which is due to Wong and $\operatorname{Li}[9,10]$, and since in our setting the characteristic roots are simple, the generally very involved expressions in Wong and Li's work become manageable.

\section{Derivation of the Model Equation}

We consider the Dirac equation in one dimension for $0 \leq x \leq L$ and periodic boundary conditions

$$
H \Psi_{E}(x)=E \Psi_{E}(x),
$$


where the Dirac operator

$$
H=-i \sigma_{x} \frac{\partial}{\partial x}+m(x) \sigma_{y}
$$

depends on Pauli matrices $\sigma_{j}$ and contains a random term $m(x)$. The latter is Gaussian distributed, independently for different sites $x$ with

$$
\langle m(x)\rangle=m_{0}, \quad\left\langle\left(m(x)-m_{0}\right)^{2}\right\rangle=2 g .
$$

$m(x) \sigma_{y}$ can be considered as a random mass. This physical problem has some interesting properties in terms of the solution $\Psi_{E}(E)[8]$. For instance, the solution is localized for $E \neq 0$ and/or $m_{0} \neq 0$, i.e., the average wavefunction $\left\langle\Psi_{E}(x)\right\rangle$ decays exponentially on a length scale $\xi\left(E, m_{0}\right)$, and $\xi\left(E, m_{0}\right)$ diverges at $E=m_{0}=0$. The Fourier transform of equation (1) in terms of time $t$ with

$$
\Psi(x, t)=\int_{-\infty}^{\infty} \Psi_{E}(x) e^{i E t} d E \quad \text { and } \quad H \Psi(x, t)=-i \frac{\partial}{\partial t} \Psi(x, t)
$$

can be considered as an initial value problem. Starting with the initial function $\Psi(x, 0)$, the time evolution is given as

$$
\Psi(x, t)=e^{i H t} \Psi(x, 0) .
$$

Assuming that the initial function $\Psi(x, 0)$ is given as a non-random function, the time-dependent solution $\Psi(x, t)$ depends on the randomness of $H$ only through the Green's function $e^{i H t}$. In order to evaluate the average wavefunction

$$
\langle\Psi(r, t)\rangle=\left\langle e^{i H t}\right\rangle \Psi(r, 0)
$$

we need the average Green's function. The latter can be associated with the Greens's function of an effective non-random, translational-invariant Hamiltonian $\mathcal{H}$, since the distribution of the random mass is translational invariant. Then the low-energy asymptotics of the average wavefunction can be determined from the spectral properties of $\mathcal{H}$. The central idea of this approach is that the Dirac equation (1) is a one-dimensional equation which can be represented as a zero-dimensional quantum problem, using the concept of second quantization.

\subsection{Effective Non-Random Hamiltonian}

For the following discussion it is convenient to use the Fourier transform of the Green's function, given by the resolvent $G(z)=(z-H)^{-1}$ such that

$$
e^{i H t}=\lim _{\epsilon \downarrow 0} \int_{-\infty}^{\infty} G(E-i \epsilon) e^{i E t} d E \quad(t>0) .
$$


$\langle G(z)\rangle$ can be formally expressed by the Greens's function of a non-linear supersymmetric theory $[6,11]$. Using the notation of Balents and Fisher [2] with $f_{s}\left(f_{s}^{\dagger}\right)$ annihilation (creation) operator of a fermion and $b_{s}\left(b_{s}^{\dagger}\right)$ annihilation (creation) operator of a boson with spin $s=\uparrow, \downarrow$, the diagonal elements of the average Green's function, for instance, read

$$
\langle G\rangle(\epsilon ; x, \uparrow ; x, \uparrow)=\operatorname{tr}\left(f_{\uparrow} f_{\uparrow}^{\dagger} e^{-L \mathcal{H}}\right),
$$

where $t r$ is the trace with respect to bosonic as well as fermionic states. The new Hamiltonian $\mathcal{H}$ is given as [2]

$$
\mathcal{H}=\epsilon\left(f^{\dagger} \cdot f+b^{\dagger} \cdot b\right)+m_{0} A-g A^{2}
$$

with Fermi (Bose) operators $f(b), f^{\dagger} \cdot f=f_{\downarrow}^{\dagger} f_{\downarrow}+f_{\uparrow}^{\dagger} f_{\uparrow}$, and

$$
A=f_{\uparrow}^{\dagger} f_{\downarrow}^{\dagger}-f_{\uparrow} f_{\downarrow}+b_{\uparrow}^{\dagger} b_{\downarrow}^{\dagger}-b_{\uparrow} b_{\downarrow} .
$$

The supersymmetric Hamiltonian is translational invariant on the interval $[0, L]$. It can be diagonalized with an appropriate unitary transformation. In contrast, the Dirac operator $H$ is not translational invariant, and to diagonalize it we would need a unitary transformation for each realization of the random $m(x)$.

\subsection{Diagonalization of the Supersymmetric Hamiltonian}

With the new operators $\Psi=(f, b), \bar{\Psi}=\left(f^{\dagger}, B^{\dagger} \sigma_{z}\right)$ and $B^{\dagger}=\left(b_{\uparrow}^{\dagger}, b_{\downarrow}\right)$ we can introduce the current

$$
\mathbf{J}_{a b}=\frac{1}{2} \bar{\Psi}_{a \alpha} \sigma_{\alpha \beta} \Psi_{b \beta}
$$

to write the Hamiltonian as

$$
\mathcal{H}=2 \epsilon J^{z}+2 m_{0} J^{x}-4 g\left(J^{x}\right)^{2}
$$

with the $x$ and $z$ components of $\mathbf{J}_{a b}, J^{x}$ and $J^{z}$. The eigenfunctions of $J^{z}$, $J^{z}|n\rangle_{0}=n|n\rangle_{0}$, form a basis set $\left\{|n\rangle_{0}\right\}(n \geq 0)$ with

$$
J^{x}|n\rangle_{0}=\frac{1}{2}\left[(n+1)|n+1\rangle_{0}-(n-1)|n-1\rangle_{0}\right] \quad(n \geq 1)
$$

and $J^{x}|0\rangle_{0}=\frac{1}{2}|1\rangle_{0}$. Then the eigenfunction $|0\rangle_{R}$ of $\mathcal{H}$ with eigenvalue $E=0$,

$$
\mathcal{H}|0\rangle_{R}=E|0\rangle_{R}=0,
$$

can be expanded in terms of the eigenfunctions of $J^{z}$ as

$$
|0\rangle_{R}=\sum_{n \geq 0} \Phi_{n}|n\rangle_{0}
$$


Equation (4) provides for the coefficients with $n \geq 0$ the recursion equation

$$
n\left[-(n+1) \Phi_{n+2}+2 n \Phi_{n}-(n-1) \Phi_{n-2}-2 M\left(\Phi_{n+1}-\Phi_{n-1}\right)+2 \omega \Phi_{n}\right]=0
$$

with the initial conditions $\Phi_{-1}=\Phi_{-2}=0$. Here we have used $M=m_{0} / 2 g$ and $\omega=\epsilon / g$. Equation (5) is called a discrete Schrödinger equation. It is the main object of the mathematical study in this paper.

\section{Analysis of the Schrödinger Equation}

Writing the fourth order equation (5) in the form

$$
\Phi_{n+2}+\frac{2 M}{n+1} \Phi_{n+1}-\frac{2(n-\omega)}{n+1} \Phi_{n}-\frac{2 M}{n+1} \Phi_{n-1}+\frac{n-1}{n+1} \Phi_{n-2}=0
$$

we see that this equation is of Poincaré type (i.e. the coefficients converge to constants as $n \rightarrow \infty)$, that the corresponding limiting equation is $\Phi_{n+2}-$ $2 \Phi_{n}+\Phi_{n-2}=0$, and that the characteristic equation $\lambda^{4}-2 \lambda^{2}+1=0$ has 1 as a fourfold root. This is a highly degenerate situation which looks, at first glance, hopeless to be accessible. On the other hand, the particular structure of equation (6) allows to rewrite this equation in the form

$$
\left(\Phi_{n+2}-\Phi_{n}\right)+\frac{2 M}{n+1}\left(\Phi_{n+1}-\Phi_{n-1}\right)+\frac{1-n}{n+1}\left(\Phi_{n}-\Phi_{n-2}\right)=\frac{-2 \omega}{n+1} \Phi_{n}
$$

which, after setting $x_{n}:=\Phi_{n}-\Phi_{n-2}$, appears as

$$
x_{n+2}+\frac{2 M}{n+1} x_{n+1}+\frac{1-n}{n+1} x_{n}=\frac{-2 \omega}{n+1} \Phi_{n} .
$$

This equation may be viewed (if $\Phi_{n}$ is considered to be known) as an inhomogeneous linear equation whose homogeneous part

$$
x_{n+2}+\frac{2 M}{n+1} x_{n+1}+\frac{1-n}{n+1} x_{n}=0
$$

is of Poincaré type, too. Moreover, the corresponding characteristic equation $\lambda^{2}-1=0$ has two simple roots, namely $\lambda_{1}=1$ and $\lambda_{2}=-1$, and this means that we have reduced the given problem to a much simpler one. But still, standard results from the Asymptotic Theory are not applicable since the two characteristic roots have the same modulus. However, employing a simplified version of the Birkhoff-Trjitzinky Theory due to Wong and Li $[9,10]$ we can tackle equation (9) and consequently (8), (7) and (6).

In order to do so we briefly describe the result which turns out to be useful for the study of (9). For more details we refer to Elaydi [7, Section 8.6]. 


\subsection{The Birkhoff-Adams Theorem}

Suppose we are given a second order linear difference equation

$$
x_{n+2}+p_{1}(n) x_{n+1}+p_{2}(n) x_{n}=0
$$

whose coefficient functions $p_{1}(n)$ and $p_{2}(n)$ have asymptotic expansions

$$
p_{1}(n) \sim \sum_{j=0}^{\infty} \frac{a_{j}}{n^{j}} \quad \text { and } \quad p_{2}(n) \sim \sum_{j=0}^{\infty} \frac{b_{j}}{n^{j}} \quad \text { as } n \rightarrow \infty
$$

with real coefficients $a_{0}, a_{1}, \ldots, b_{0}, b_{1}, \ldots$. The limiting equation of (10) is $x_{n+1}+a_{0} x_{n+1}+b_{0} x_{n}=0$, and the corresponding characteristic roots are

$$
\lambda_{1 / 2}=-\frac{a_{0}}{2} \pm \sqrt{\frac{a_{0}^{2}}{4}-b_{0}^{2}} .
$$

If $\lambda_{1} \neq \lambda_{2}$, then equation (10) has two linearly independent solutions $x_{1, n}$ and $x_{2, n}$ of the form

$$
x_{i, n} \sim \lambda_{i}^{n} n^{\alpha_{i}} \sum_{r=0}^{\infty} \frac{c_{i}(r)}{n^{r}}, \quad i=1,2, \quad \text { as } \quad n \rightarrow \infty
$$

where the $\alpha_{i}$ and $c_{i}(r)$ are given as follows: For the $\alpha_{i}$ we have the explicit formula

$$
\alpha_{i}=\frac{a_{1} \lambda_{i}+b_{1}}{a_{0} \lambda_{i}+2 b_{0}}, \quad i=1,2,
$$

and for the $c_{i}(r)$ we have the following recursion: $c_{i}(0)=1$ and

$$
\sum_{j=0}^{s-1}\left[\lambda_{i}^{2} 2^{s-j}\left(\begin{array}{c}
\alpha_{i}-j \\
s-j
\end{array}\right)+\lambda_{i} \sum_{r=j}^{s}\left(\begin{array}{c}
\alpha_{i}-j \\
r-j
\end{array}\right) a_{s-r}+b_{s-j}\right]=0, \quad i=1,2 .
$$

This in particular implies

$$
c_{i}(1)=\frac{-2 \lambda_{i}^{2} \alpha_{i}\left(\alpha_{i}-1\right)-\lambda_{i}\left(a_{2}+\lambda_{i} a_{1}+\alpha_{i}\left(\alpha_{i}-1\right) a_{0} / 2\right)-b_{2}}{2 \lambda_{i}^{2}\left(\alpha_{i}-1\right)+\lambda_{i}\left(a_{1}+\left(\lambda_{i}-1\right) a_{0}\right)+b_{1}}, \quad i=1,2 .
$$

The case $\lambda_{1}=\lambda_{2}$, by the way, is more involved (see Elaydi [7, Theorem 8.36]), but it is not needed in this paper.

\subsection{The Schrödinger Equation with $\omega=0$}

In order to apply the result described in the previous subsection to the homogeneous equation (9) (which represents the case $\omega=0$ in equation (6)) we have to put the coefficients $\frac{2 M}{n+1}$ and $\frac{1-n}{n+1}$ appearing in this equation in the required form. Using the relation $\frac{1}{n+1}=\frac{1}{n}-\frac{1}{n^{2}}+O\left(\frac{1}{n^{2}}\right)$ as $n \rightarrow \infty$ we get

$$
\frac{2 M}{n+1}=\frac{2 M}{n}-\frac{2 M}{n^{2}}+O\left(\frac{1}{n^{2}}\right) \text { and } \frac{1-n}{n+1}=-1+\frac{2}{n}-\frac{2}{n^{2}}+O\left(\frac{1}{n^{2}}\right)
$$


as $n \rightarrow \infty$, and this implies

$$
a_{0}=0, \quad a_{1}=2 M, \quad a_{2}=-2 M ; \quad b_{0}=-1, \quad b_{1}=2, \quad b_{2}=-2 .
$$

Recalling that $\lambda_{1}=1$ and $\lambda_{2}=-1$ we therefore get

$$
\begin{array}{ll}
\alpha_{1}=-M-1, & c_{1}(1)=M^{2}+3 M+1, \\
\alpha_{2}=M-1, & c_{2}(1)=M^{2}-M+1 .
\end{array}
$$

Thus, by the Birkhoff-Adams Theorem, equation (9) has a fundamental set of solutions of the form

$$
\begin{aligned}
& x_{1, n} \sim n^{-M-1}\left[1+\frac{M^{2}+3 M+1}{n}+O\left(n^{-2}\right)\right] \\
& x_{2, n} \sim(-1)^{n} n^{M-1}\left[1+\frac{M^{2}-M+1}{n}+O\left(n^{-2}\right)\right] .
\end{aligned}
$$

Going back to the original equation (7) with $\omega=0$ we get the two relations

$$
\begin{aligned}
& \Phi_{n+2}-\Phi_{n} \sim x_{1, n} \quad \text { as } \quad n \rightarrow \infty \\
& \Phi_{n+2}-\Phi_{n} \sim x_{2, n} \quad \text { as } \quad n \rightarrow \infty .
\end{aligned}
$$

Using the two solutions $y_{1, n}=1$ and $y_{2, n}=(-1)^{n}$ of the difference equation $\Phi_{n+2}-\Phi_{n}=0$, the variation of constants formula stipulates that a particular solution of (13) or (14) can be written in the form

$$
\Phi_{n}=u_{1, n} y_{1, n}+u_{2, n} y_{2, n}
$$

where

$$
u_{1, n}=\sum_{r=0}^{n-1} \frac{-g(r) y_{2, r+1}}{W(r+1)}, \quad u_{2, n}=\sum_{r=0}^{n-1} \frac{g(r) y_{1, r+1}}{W(r+1)}
$$

with $g(n)$ being $x_{1, n}$ or $x_{2, n}$, respectively, and $W(r+1)$ being the Casoratian

$$
W(r+1)=y_{1, r+1} y_{2, r+2}-y_{1, r+2} y_{2, r+1}=(-1)^{r+2}-(-1)^{r+1}=2(-1)^{r} .
$$

For the asymptotic relation (13) we thus get

$$
u_{1, n}=\frac{1}{2} \sum_{r=0}^{n-1} x_{1, r} \quad \text { and } \quad u_{2, n}=\frac{1}{2} \sum_{r=0}^{n-1}(-1)^{r} x_{1, r},
$$

and this yields for (13) the particular solution

$$
\Phi_{n}^{*} \sim \frac{1}{2} \sum_{r=0}^{n-1}\left[1+(-1)^{n+r}\right] x_{1, r}
$$

where $x_{1, r}$ is given in (11). Using the particular form of $y_{1, n}$ and $y_{2, n}$, for arbitrary real constants $\gamma_{1}, \gamma_{2}$ we then get the following two solutions of (13):

$$
\Phi_{1, n} \sim \Phi_{n}^{*}+\gamma_{1} \text { and } \Phi_{2, n} \sim \Phi_{n}^{*}+(-1)^{n} \gamma_{2} .
$$


With the same arguments as before we get two solutions of (14), namely

$$
\Phi_{3, n} \sim \Phi_{n}^{* *}+\gamma_{3} \quad \text { and } \quad \Phi_{4, n} \sim \Phi_{n}^{* *}+(-1)^{n} \gamma_{4},
$$

where $\gamma_{3}, \gamma_{4}$ are arbitrary real constants and $\Phi_{n}^{* *}$ is the particular solution

$$
\Phi_{n}^{* *} \sim \frac{1}{2} \sum_{r=0}^{n-1}\left[1+(-1)^{n+r}\right] x_{2, r}
$$

of (14) with $x_{2, r}$ given in (12). Thus, altogether we have obtained the four linearly independent solutions $\Phi_{1, n}, \ldots, \Phi_{4, n}$ of the special Schrödinger equation (6) with $\omega=0$.

\subsection{The Full Schrödinger Equation}

We now deal with the full equation (6). Setting again $x_{n}:=\Phi_{n}-\Phi_{n-2}$ we obtain the equation

$$
x_{n+2}+\frac{2 M}{n+1} x_{n+1}+\frac{1-n}{n+1} x_{n}=\frac{-2 \omega}{n+1} \Phi_{n}
$$

which we look at as an inhomogeneous equation for the $x_{n}$. The corresponding homogeneous part

$$
x_{n+2}+\frac{2 M}{n+1} x_{n+1}+\frac{1-n}{n+1} x_{n}=0
$$

has been investigated in the previous section. Using the two linearly independent solutions $x_{1, n}$ and $x_{2, n}$ of (16) (see (11) and (12)) and going back to equation (6) be resubstituting $x_{n}=\Phi_{n}-\Phi_{n-2}$ we get the two relations $\Phi_{n+2}-\Phi_{n} \sim x_{1, n}-\frac{2 \omega}{n+1} \Phi_{n}$ and $\Phi_{n+2}-\Phi_{n} \sim x_{2, n}-\frac{2 \omega}{n+1} \Phi_{n}$, or equivalently,

$$
\begin{aligned}
& \Phi_{n+2}+\left(\frac{2 \omega}{n+1}-1\right) \Phi_{n} \sim x_{1, n} \\
& \Phi_{n+2}+\left(\frac{2 \omega}{n+1}-1\right) \Phi_{n} \sim x_{2, n} .
\end{aligned}
$$

In order to find the solutions of these relations we first solve the associated homogeneous equation

$$
\Phi_{n+2}+\left(\frac{2 \omega}{n+1}-1\right) \Phi_{n}=0
$$

This equation is of Poincaré type, and the corresponding characteristic equation $\lambda^{2}-1=0$ has the two roots $\lambda_{1}=1$ and $\lambda_{2}=-1$. Using the relation

$$
\frac{2 \omega}{n+1}-1=-1+\frac{2 \omega}{n}-\frac{2 \omega}{n^{2}}+O\left(\frac{1}{n^{2}}\right) \quad \text { as } \quad n \rightarrow \infty
$$


and employing the notation associated with the Birkhoff-Adams Theorem we get

$$
a_{0}=a_{1}=a_{2}=0 ; \quad b_{0}=-1, \quad b_{1}=2 \omega, \quad b_{2}=-2 \omega,
$$

and this implies

$$
\alpha_{1}=\alpha_{2}=-\omega \quad \text { and } \quad c_{1}(1)=c_{2}(1)=\omega^{2} .
$$

Applying the Birkhoff-Adams Theorem to equation (19) we therefore get two linearly independent solutions

$$
\begin{aligned}
& z_{1, n} \sim n^{-\omega}\left[1+\frac{\omega^{2}}{n}+O\left(n^{-2}\right)\right], \\
& z_{2, n} \sim(-1)^{n} n^{-\omega}\left[1+\frac{\omega^{2}}{n}+O\left(n^{-2}\right)\right] .
\end{aligned}
$$

For the inhomogeneous relations (17) and (18) we can then find particular solutions in the form

$$
\Psi_{n}=v_{1, n} z_{1, n}+v_{2, n} z_{2, n}
$$

where

$$
v_{1, n}=\sum_{r=0}^{n-1} \frac{-g(r) z_{2, r+1}}{V(r+1)}, \quad v_{2, n}=\sum_{r=0}^{n-1} \frac{g(r) z_{1, r+1}}{V(r+1)}
$$

with $g(n)$ being $x_{1, n}$ or $x_{2, n}$, respectively, and $V(r+1)$ being the Casoratian

$$
V(r+1)=z_{1, r+1} z_{2, r+2}-z_{1, r+2} z_{2, r+1} .
$$

Choosing $g(r)=x_{1, n}$, this allows to compute a particular solution $\Psi_{n}^{*}$ for the asymptotic relation (17), and with this we get two linearly independent solutions of (17) of the form

$$
\Psi_{1, n} \sim \Psi_{n}^{*}+\gamma_{1} z_{1, n} \quad \text { and } \quad \Psi_{2, n} \sim \Psi_{n}^{*}+\gamma_{2} z_{2, n}
$$

with real parameters $\gamma_{1}$ and $\gamma_{2}$. Accordingly, we get two linearly independent solutions of (18) in the form

$$
\Psi_{3, n} \sim \Psi_{n}^{* *}+\gamma_{3} z_{1, n} \quad \text { and } \quad \Psi_{4, n} \sim \Psi_{n}^{* *}+\gamma_{4} z_{2, n}
$$

where $\gamma_{3}$ and $\gamma_{4}$ are real parameters and $\Psi_{n}^{* *}$ is a particular solution of (18) which can be obtained from (22) and (23) by choosing $g(r)=x_{2, n}$. In summary, we have obtained the four linearly independent solutions $\Psi_{1, n}, \ldots, \Psi_{4, n}$ of the Schrödinger equation (6).

\section{Conclusion}

The purpose of this paper is to demonstrate how a physical problem of current interest, the motion of a relativistic quantum particle with random mass, 
can be reduced to a problem on difference equations, and how the resulting difference equation, a highly critical equation of Poincaré type, can be solved. While the derivation of the difference equation is worked out in detail, the

limited size of this paper allows to present its solution only in its principal steps. More details will follow in a forthcoming paper.

\section{References}

[1] Adams, C. R., On the irregular cases of linear ordinary difference equations, Trans. Amer. Math. Soc. 30 (1928), 507-541.

[2] Balents, L. and Fisher, M. P. A., Delocalization transition via supersymmetry in one dimension, Phys. Rev. B 56 (1997), 12970-12991.

[3] Birkhoff, G.D., General theory of linear difference equations, Trans. Amre. Math. Soc. 12 (1911), 243-284.

[4] Birkhoff, G. D., Formal theory of irregular linear difference equatiions, Acta Math. 54 (1930), 205-246.

[5] Birkhoff, G. D. and Trjitzinsky, W. J., Analytic theory of singular difference equations, Acta Math. 60 (1932), 1-89.

[6] Efetov, K. B., Supersymmetry and theory of disordered metals, Adv. Phys. 32 (1983), 53-127.

[7] Elaydi, S. N., An Introduction to Difference Equations, 2nd Ed., Springer, New York 1999.

[8] Takeda, K., Tsurumaru, T., Ichinose, I. and Kimura, M., Localized and extended states in one-dimensional disordered system: Random mass Dirac fermions, Nucl. Phys. B 556 (1999), 545-562.

[9] Wong, R. and Li, H., Asymptotic expansions for second order linear difference equations, J. Comput. Appl. Math. 41 (1992), 65-94.

[10] Wong, R. and Li, H., Asymptotic expansions for second order linear difference equations II, Studies Appl. Math. 87 (1992), 289-324.

[11] Ziegler, K., Disordered system with $\mathrm{n}$ orbitals per site: Langrange formulation without replica trick, and scaling law for the density of states, Z. Phys. B 48 (1982), 293-304. 